\title{
Fractal geometry in an expanding, one-dimensional, Newtonian universe
}

\author{
Bruce N. Miller* \\ Department of Physics and Astronomy, Texas Christian University, Fort Worth, Texas 76129, USA \\ Jean-Louis Rouet \\ Institut des Sciences de la Terre d'Orléans, (ISTO)_UMR 6113 CNRS/Université d'Orléans, 1A, rue de la Férollerie, \\ 45071 Orléans Cedex 2, France \\ and Laboratoire de Mathématique, Applications et Physique Mathématique-UMR 6628 CNRS/Université d'Orléans, \\ UFR des Sciences, F-45067 Orléans Cedex 2, France \\ Emmanuel Le Guirriec \\ Laboratoire de Mathématique, Applications et Physique Mathématique-UMR 6628 CNRS/Université d'Orléans, \\ UFR des Sciences, F-45067 Orléans Cedex 2, France
}

(Received 18 December 2006; revised manuscript received 19 June 2007; published 14 September 2007)

\begin{abstract}
Observations of galaxies over large distances reveal the possibility of a fractal distribution of their positions. The source of fractal behavior is the lack of a length scale in the two body gravitational interaction. However, even with new, larger, sample sizes from recent surveys, it is difficult to extract information concerning fractal properties with confidence. Similarly, three-dimensional $N$-body simulations with a billion particles only provide a thousand particles per dimension, far too small for accurate conclusions. With one-dimensional models these limitations can be overcome by carrying out simulations with on the order of a quarter of a million particles without compromising the computation of the gravitational force. Here the multifractal properties of two of these models that incorporate different features of the dynamical equations governing the evolution of a matter dominated universe are compared. For each model at least two scaling regions are identified. By employing criteria from dynamical systems theory it is shown that only one of them can be geometrically significant. The results share important similarities with galaxy observations, such as hierarchical clustering and apparent bifractal geometry. They also provide insights concerning possible constraints on length and time scales for fractal structure. They clearly demonstrate that fractal geometry evolves in the $\mu$ (position, velocity) space. The observed patterns are simply a shadow (projection) of higher-dimensional structure.
\end{abstract}

DOI: 10.1103/PhysRevE.76.036705 PACS number(s): 05.10.-a, 05.45.-a, 98.65. $-\mathrm{r}, 98.80 .-\mathrm{k}$

\section{INTRODUCTION}

Starting with the work of Vaucouleurs [1], questions have been posed about the geometric properties of the distribution of matter in the universe. Necessary for all modern cosmologies are the assumptions of homogeneity and isotropy of the mass distribution on large length scales [2]. However, observations have shown the existence of very large structures such as superclusters and voids [3]. Moreover, as technology has advanced, so has the length scale of the largest observed structures. (For a recent review see Jones et al. [4].) It was proposed by Mandelbrot [5], based on the work of Peebles [2], that the matter distribution in the universe is fractal. Support for this conjecture came primarily from the computation of the pair correlation function for the positions of galaxies, as well as from direct observation. The fact that the correlation function was well represented by a power law in the intergalactic distance seemed to support the fractal conjecture. In fact, in the past it has been argued by Pietronero and colleagues that the universe may even be fractal on all scales $[4,6]$. Of course, if this were true it would wreak havoc with the conclusions of cosmological theory.

McCauley has looked at this issue from a few different perspectives. He points out that since the power law behavior

\footnotetext{
*b.miller@tcu.edu; URL: http://personal.tcu.edu/ bmiller
}

of the correlation function is only quantitatively correct over a finite length scale, the universe could not be a simple (mono) fractal [7]. Then the logical next question is whether or not the matter distribution is multifractal. By considering counts in cells, Balian and Schaeffer hypothesized some time ago that the distribution of matter is approximately bifractal, i.e., a superposition of fractals with two distinct scaling laws [8]. They conjectured that the overdense clusters and underdense voids had distinctive geometries, which were the source of the different dimensions. McCauley has also addressed the possibility of inhomogeneity. Working with Martin Kerscher, he investigated whether the universe has multifractal geometry. His approach was to examine the pointwise dimension [9] by looking for local scaling of the density around individual galaxies in two catalogues. Their conclusion was that, even if local scaling were present, there are large fluctuations in the scaling law. Moreover, the sample sizes were not sufficient to enable the extraction of good local scaling exponents. Although larger galaxy catalogues have become available since their analysis, they do not meet the restrictive criteria of sufficient size, as well as uniform extension in all directions, necessary to measure local dimensions [4]. Their final conclusion was that, while the geometry of the observed universe is certainly not monofractal, it was not possible to irrefutably conclude whether it is multifractal, or whether the assumptions of homogeneity and isotropy prevail at large length scales [7]. 
If we are not able to determine the geometry of the universe from current observations, then we need to turn to theory and simulation. For the most part, we are concerned with the evolution of matter after the period of recombination. Consequently, the Hubble expansion has slowed down sufficiently that Newtonian dynamics is applicable, at least within a finite region of space [10]. A number of theoretical approaches to the computation of fractal dimensions have been investigated. Each of them is predicated on some external assumption, which is not yet verified. For example, de Vega et al. assume that the universe is close to a thermodynamic critical point [11], while Grujic explores a field theory where vacuum energy predominates over inert matter, and the latter is assumed to have a fractal structuring [12]. An examination of the recent literature reveals that theory has not converged on a compelling and uniformly accepted theory of fractal structure in the universe.

In the last few decades dynamical $N$-body simulation of cold dark matter (CDM) has experienced rapid advances due to improvements in both algorithms and technology $[13,14]$. It is now possible to carry out gravitational $N$-body simulations in three space dimensions with upwards of $10^{9}$ point mass particles. However, in order to employ simulation methods for systems evolving over cosmological time, it is necessary to compromise the representation of the gravitational interaction over both long and short length scales. For example, tree methods are frequently employed for large separations, typically the gravitational field is computed from a grid, and a short range cutoff is employed to control the singularity in the Newtonian pair potential $[13,14]$. Unfortunately, even if the simulations were perfect, a system of even $10^{9}$ particles provides only $10^{3}$ particles per dimension and would thus be insufficient to investigate the fractal geometry with confidence.

As a logical consequence of these difficulties it was natural that physicists would look to lower-dimensional models for insight. Although this sacrifices the correct dynamics, it provides an arena where accurate computations with large numbers of particles can be carried out for significant cosmological time. It is hoped that insights gained from making this trade-off are beneficial. In one dimension, Newtonian gravity corresponds to a system of infinitesimally thin, parallel, mass sheets of infinite spatial extent, each moving in the direction perpendicular to its surface. Because the acceleration of each particle is constant between crossings, the equations of motion can be integrated exactly. Therefore it is not necessary to use numerical integration to follow the trajectory in phase space. As a consequence it was possible to study the system dynamics on the earliest computers and it can be considered the gravitational analogue of the FermiPasta-Ulam system [15]. It was first employed by Lecar and Cohen to investigate the relaxation of an $\mathrm{N}$-body gravitational system [16]. Although it was first thought that the $N$-body system would reach equilibrium with a relaxation time proportional to $N^{2}$ [17], this was not borne out by simulations [18]. Partially because of their reluctance to reach equilibrium, both single and two component versions of the system have been studied exhaustively in recent years [19-24]. Most recently, it was demonstrated that, for short times and special initial conditions, the system evolution can be modeled by an exactly integrable system [25]. To gain insight into the dynamics of core collapse, one-dimensional systems consisting of spherical mass shells have also been investigated. There it was found that, if the singularity of the Newtonian gravitational interaction is screened, then both irrotational [26,27] and rotating [28-30] systems can exist in different thermodynamics phases. Moreover, if angular momentum exchange between shells is permitted, the system will initially be attracted by metastable Vlasov states in the $\mu$ space, but will eventually undergo core collapse [31]. A different model for studying collapse, in which a Gaussian noise term has been added to the deterministic gravitational interaction, has recently been introduced by Chavanis [32]. The stationary solution of the one-dimensional version of this model has been proven to be stable with respect to symmetric fluctuations in the large friction limit [33]. Under different conditions, density singularities (shocks) can occur and the system has been shown to be equivalent to a fluid obeying Burger's equation [33] (see below).

Since there is no curvature in a $(1+1)$-dimensional gravitational system, we cannot expect to obtain equations of motion directly from general relativity [34]. Then a question arises concerning the inclusion of the Hubble flow into the dynamical formulation. This has been addressed in two ways: In the earliest, carried out by Rouet and Feix (RF), the scale function was directly inserted into the one-dimensional dynamics $[35,36]$. Alternatively, starting with the usual threedimensional equations of motion and embedding a stratified mass distribution, Fanelli and Aurell obtained a similar set of coupled differential equations for the evolution of the system in phase space [37]. While the approaches are different, from the standpoint of mathematics the two models are very similar and differ only in the values of a single fixed parameter, the effective friction constant. Following Fanelli [37], we refer to the former as the RF model and the latter as the quintic, or $Q$, model.

By introducing scaling in both position and time, in each model autonomous equations of motion are obtained in the comoving frame. In addition to the contribution for the gravitational field, there is now a background term, corresponding to a constant negative mass distribution, and a linear friction term. By eliminating the friction term, a Hamiltonian version can also be constructed. At least three other one dimensional models have also been investigated to gain insight into gravitational clustering, one consisting of Newtonian mass sheets, which stick together whenever they cross [38], one evolved by directly integrating the Zeldovich equations $[39,40]$, and the continuous system satisfying Burger's equation [41]. In addition, fractal behavior has been studied in the autonomous one-dimensional system where there is no background Hubble flow $[42,43]$.

In dynamical simulations, both the RF and quintic models clearly manifest the development of hierarchical clustering in both configuration and $\mu$ space (the projection of the phase space on the position-velocity plane). In common with the observation of galaxy positions, as time evolves both dense clusters and relatively empty regions (voids) develop. In their seminal work, by computing the box-counting dimension for the RF model, Rouet and Feix were able to directly demonstrate the formation of fractal structure $[35,36]$. They 
found a value of about 0.6 for the box counting dimension of the well evolved mass points in the configuration space, indicating the formation of a robust fractal geometry. In a later work, Miller and Rouet investigated the generalized dimension of the RF model [44]. In common with the analysis of galaxy observations by Balian and Schaeffer [8] they found evidence for bifractal geometry. Although they did not compute actual dimensions, later Fanelli et al. also found a suggestion of bifractal behavior in position in the model without friction [45]. It is then not surprising that the autonomous, isolated, gravitational system that does not incorporate the Hubble flow also manifests fractal behavior for short times as long as the virial ratio realized by the initial conditions is very small, i.e., as long as the system is initially cold $[42,43]$. In addition, in the one-dimensional model of turbulence governed by Burger's equation, the formation of shocks has the appearance of density singularities that are similar to the clusters found in the RF and quintic model. A type of bifractal geometry has also been demonstrated for this system [41].

Our earlier paper [44] should be regarded as a preliminary investigation of the RF model that raised a number of questions concerning the nature of the scaling regions in position. In the current work, hierarchical structure formation in two related models that were proposed by other groups is carefully compared, and a number of important issues are resolved. In particular, the appearance of two scaling ranges, the counterintuitive identification of the larger scaling range as the physically relevant one, and the likely source of the smaller range are now, for the most part, understood. In addition, the fractal analysis is carried out in the higherdimensional $\mu$ (position, velocity) space as well as on the real line. It is demonstrated that the basic structures are formed there, and that the fractal-like features of the distribution in position are a projection of the higher-dimensional geometry. Moreover it is demonstrated that, even when there is no effective dissipation in the comoving frame, mass in the underdense voids is attracted by parallel, linear, segments in the $\mu$ space.

Below we present the results of our recent investigation of multifractal properties of the quintic model and the model without friction. In Sec. II we will first describe the systems. Then we will give a straightforward derivation of the equations of motion and explain how they differ from the other models mentioned above. We will also apply the VlasovPoisson equations in the comoving frame to prove that low density matter is attracted to parallel, linear, segments in the $\mu$ space. In Sec. III we will explain how the simulations were carried out and describe their qualitative features. In Sec. IV we define the generalized dimension and other fractal measures and present our approach for computing them. In Sec. $\mathrm{V}$ we will present the results of the multifractal analysis. Finally, conclusions will be presented in Sec. VI.

\section{DESCRIPTION OF THE SYSTEM}

We are interested in the development of density fluctuations following the time of recombination, so that electromagnetic forces can be ignored. For that and more recent epochs, the Hubble expansion has slowed sufficiently that
Newtonian dynamics provides an adequate representation of the motion in a finite region [10]. Then, in a $(3+1)$ dimensional universe, the Newtonian equations governing a mass point are simply

$$
\frac{d \mathbf{r}}{d t}=\mathbf{v}, \quad \frac{d \mathbf{v}}{d t}=\mathbf{E}_{g}(\mathbf{r}, t),
$$

where, here, $\mathbf{E}_{g}(\mathbf{r}, t)$ is the gravitational field. To follow the motion in a frame of reference where the average density remains constant, i.e., the comoving frame, we introduce the scale factor $A(t)$ for a matter dominated universe [2] and transform to a new space coordinate which scales the distance according to $A(t)$. Writing $\mathbf{r}=A(t) \mathbf{x}$ we obtain

$$
\frac{d^{2} \mathbf{x}}{d t^{2}}+\frac{2}{A} \frac{d A}{d t} \frac{d \mathbf{x}}{d t}+\frac{1}{A} \frac{d^{2} A}{d t^{2}} \mathbf{x}=\frac{1}{A^{3}} \mathbf{E}_{g}(\mathbf{x}, t),
$$

where, in the above we have taken advantage of the inverse square dependence of the gravitational field to write $\mathbf{E}_{g}(\mathbf{x}, t)=\frac{1}{A^{2}} \mathbf{E}_{g}(\mathbf{r}, t)$, where the functional dependence is preserved. In a matter dominated (Einstein-de Sitter) universe we find that

$$
A(t)=\left(\frac{t}{t_{0}}\right)^{2 / 3}, \quad \rho_{b}(t)=\left(6 \pi G t^{2}\right)^{-1},
$$

where $t_{0}$ is some arbitrary initial time corresponding, say, to the epoch of recombination, $G$ is the universal gravitational constant, and $\rho_{b}(t)$ is the average, uniform, density frequently referred to as the background density. These results can be obtained directly from Eq. (2) by noting that if the density is uniform, so that all matter is moving with the Hubble flow, the first two terms in Eq. (2) vanish whereas the third term (times $A$ ) must be equated to the gravitational field resulting from the uniformly distributed mass contained within a sphere of radius $A|\mathbf{x}|$. Then the third term of Eq. (2) is simply the contribution arising by subtracting the field due to the background density from the sphere [2]. Noting that $A^{3} \rho_{b}(t)=\rho_{b}\left(t_{0}\right)$ forces the result. Alternatively, also for the case of uniform density, taking the divergence of each side of Eq. (2) and asserting the Poisson equation forces the same result. Thus the Friedman scaling is consistent with the coupling of a uniform Hubble flow with Newtonian dynamics [2].

For computational purposes, it is useful to obtain autonomous equations of motion that do not depend explicitly on the time. This can be effectively accomplished $[35,36]$ by transforming the time coordinate according to

$$
d t=B(t) d \tau, \quad B(t)=\frac{t}{t_{0}},
$$

yielding the autonomous equations

$$
\frac{d^{2} \mathbf{x}}{d \tau^{2}}+\frac{1}{3 t_{0}} \frac{d \mathbf{x}}{d \tau}-\frac{2}{9 t_{0}^{2}} \mathbf{x}=\mathbf{E}_{g}(\mathbf{x})
$$

For the Newtonian dynamics considered here, it is necessary to confine our attention to a bounded spatial region $\Omega$. It is customary to choose a cube for $\Omega$ and assume periodic boundary conditions. Thus our equations correspond to a dis- 
sipative dynamical system in the comoving frame with friction constant $1 / 3 t_{0}$ and with forces arising from fluctuations in the local density with respect to a uniform background.

For the special case of the stratified mass distribution induced by the one-dimensional system, the local density at time $t$ is given by

$$
\rho(x, t)=\sum m_{j}(t) \delta\left(x-x_{j}\right),
$$

where $m_{j}(t)$ is the mass per unit area of the $j$ th sheet and, from symmetry, the gravitational field only has a component in the $x$ direction. From Gauss' law we know that the field due to a single mass sheet is constant, so the field experienced by one of the sheets is proportional to the difference between the mass density on each side. For the special case of equal masses $m_{j}(t)=m(t)$, the correct form of the gravitational field occurring on the right hand side of Eq. (5) at the location of particle $i$ is then

$$
E_{g}\left(x_{i}\right)=2 \pi m\left(t_{0}\right) G\left[N_{R, i}-N_{L, i}\right],
$$

since we already implicitly accounted for the fact that $m_{j}(t)$ $=m\left(t_{0}\right) / A^{2}$ in Eq. (5) and $N_{R, i}\left(N_{L, i}\right)$ is the number of sheets on the right (left) of particle $j$. The equations are further simplified by establishing the connection between $m\left(t_{0}\right)$ and the background density at the initial time $\rho_{b}\left(t_{0}\right)$. Let us assume that we have $2 N$ particles (sheets) confined within a slab with width $2 L$, i.e., $-L<x<L$. Then

$$
\rho_{b}\left(t_{0}\right)=\left(6 \pi G t_{0}^{2}\right)^{-1}=\left(\frac{N}{L}\right) m\left(t_{0}\right),
$$

we may express the field by

$$
E_{g}\left(x_{i}\right)=2 \pi m\left(t_{0}\right) G\left[N_{R, i}-N_{L, i}\right]=\frac{2}{3 t_{0}^{2}}\left(\frac{L}{N}\right)\left[N_{R, i}-N_{L, i}\right],
$$

and the equations of motion for a particle in the system now read

$$
\frac{d^{2} x_{i}}{d \tau^{2}}+\frac{1}{3 t_{0}} \frac{d x_{i}}{d \tau}-\frac{2}{9 t_{0}^{2}} x_{i}=\frac{2}{3 t_{0}^{2}}\left(\frac{L}{N}\right)\left[N_{R, i}-N_{L, i}\right] .
$$

It is convenient to refer to Jeans theory for the final choice of units of time and length [46],

$$
\begin{gathered}
T_{J}=\omega_{J}^{-1}=(4 \pi G \rho)^{-1 / 2}=\sqrt{\frac{3}{2}} t_{0}, \\
\lambda_{J}=2 \pi \sqrt{3} \frac{\sigma_{v}}{\omega_{J}}=2 \pi \frac{a}{\omega_{J}},
\end{gathered}
$$

for a one-dimensional system [35,36], where the distribution of initial velocities is uniform on $[-a, a]$ and its variance, $\sigma_{v}^{2}=a^{2} / 3$. Then, with the further requirement that $L=n \lambda_{j}, 0$ $<n<N$, in these new units, our equations are

$$
\frac{d^{2} x_{i}}{d \tau^{2}}+\frac{1}{\sqrt{6}} \frac{d x_{i}}{d \tau}-\frac{1}{3} x_{i}=\left(\frac{n}{N}\right)\left[N_{R, i}-N_{L, i}\right] .
$$

There is still a final issue that we have to address. If we assume for the moment that the sheets are uniformly distrib- uted and moving with the Hubble flow, then the first two terms above are zero. Unfortunately, if we take the average over each remaining term, we find that they differ by a factor of three. This seeming discrepancy arises because we started with spherical symmetry about an arbitrary point for the Hubble flow and are now imposing axial symmetry. If we imagine that we are in a local region with a stratified geometry, the symmetry is different and this has to be reflected in the background term. This apparent discrepancy can be rectified by multiplying the term in $x_{i}$ by three. Our final equations of evolution are then

$$
\frac{d^{2} x_{i}}{d \tau^{2}}+\frac{1}{\sqrt{6}} \frac{d x_{i}}{d \tau}-x_{i}=\left(\frac{n}{N}\right)\left[N_{R, i}-N_{L, i}\right]
$$

The description is completed by assuming that the system satisfies periodic boundary conditions on the interval $2 L$, i.e., when a particle leaves the primitive cell defined by $-L<x$ $<L$ on the right, it reenters at the left hand boundary with the identical velocity. Note that, in computing the field, we do not attempt to include contributions from the images of $x_{i}$ outside of the primitive cell. In our simulations we made the further assumption of symmetry about the origin. With this stipulation the periodic boundary conditions are equivalent to reflecting boundary conditions at $x=0, L$. In fact, for the times of interest, we anticipate that the general behavior of the system will be independent of the details of the boundary conditions.

By embedding the stratified system into a region of threedimensional Euclidean space we are changing the local symmetry, so we have to adjust the local background density. In contrast, the RF model is obtained from the reverse sequence where one first restricts the geometry to $1+1$ dimensions and then introduces the transformation to the comoving frame. In this approach the derivation is more straightforward; it is not necessary to make the adjustment in the coefficient of $x_{i}$ as we did here to obtain the correct background contribution $[35,36,44]$. This is quickly seen by noting that the divergence of $\mathbf{x}$ is three times greater than the divergence of $x \hat{\mathbf{x}}$, which one would obtain by directly starting with the onedimensional model. However, in the RF model, the coefficient of the first derivative term (the friction constant) is $1 / \sqrt{2}$ instead of $1 / \sqrt{6}$. This simply illustrates that, since there is no curvature in a $(1+1)$-dimensional universe, there is a degree of arbitrariness in choosing the final model. It cannot be obtained solely from general relativity. For a discussion of this point, see Mann et al. [34]. A Hamiltonian version can also be considered by setting the friction constant to zero. In their earlier work, using the linearized Vlasov-Poisson equations, Rouet and Feix carried out a stability analysis of the model without friction. They determined that the system followed the expected behavior, i.e., when the system size is greater than the Jean's length, instability occurs and clustering becomes possible $[35,36]$. Of course, when the friction term is not present, both the $Q$ and RF models are identical. Then, with the assumption that the friction term will not have a large influence on short-time linear stability, the analysis of the Hamiltonian version applies equally to each version. 
The Vlasov-Poisson limit for the system is obtained by letting $N \rightarrow \infty$ and $m \rightarrow 0$ while constraining the density and energy (at a given time). Then, in the comoving frame, the system is represented by a fluid in the $\mu$ space. Let $f(x, v ; t)$ represent the normalized distribution of mass in the $(x, v)$ phase plane at time $t$. From mass conservation, $f$ satisfies the continuity equation

$$
\frac{\partial f}{\partial t}+v \frac{\partial f}{\partial x}+\frac{\partial a f}{\partial v}=0, \quad a=-\gamma v-\frac{\partial \phi_{T}(x)}{\partial x},
$$

that can also be derived using the identical scaling as above. In Eq. (14), $a(x, v)$ is the local acceleration and $\phi_{T}$ is the potential function induced by the total density including the effective negative background density $-\rho_{b}$. Note that both $a(x, v)$ and $\phi_{T}$ are linear functionals of $f(x, v ; t)$. Coupling Eq. (14) with the Poisson equation yields the complete Vlasov-Poisson system governing the evolution of $f(x, v ; t)$. Depending on the final choice of the friction constant $\gamma$ the continuum limit of either the quintic or RF model can be represented by Eq. (14).

Note that an exact solution is

$$
f(x, v, t)=\frac{\left|\rho_{b}\right|}{\sqrt{2 \pi \sigma^{2}}} \exp \left(-\frac{v^{2}}{2 \sigma^{2}}\right), \quad \sigma(t)=\sigma_{0} \exp (-\gamma t) .
$$

Using dynamical simulation, we will see that it is extremely unstable when the system size exceeds the Jeans' length at the initial time.

Useful information can be had without constructing an explicit solution. For example, we quickly find that the system energy decreases at a rate proportional to the kinetic energy. The Tsallis entropy is defined by

$$
S_{q}=\frac{1-\iint f^{q} d x d v}{q-1}, \quad \iint f d x d v=1 .
$$

In the limit $q \rightarrow 1, S_{q}$ reduces to the usual Gibbs entropy $S_{1}$ $=-\iint f \ln f d x d v$. By asserting the Vlasov-Poisson evolution, we find that the Boltzmann-Gibbs entropy $S_{1}$ decreases at the constant rate $-2 \gamma$ while, for $q>1, S_{q}$ decreases exponentially in time. This tells us that the mass is being concentrated in regions of decreasing area of the phase plane, suggesting the development of structure. These properties are immediately evident for the trivial solution given above. By imposing a Euclidean metric in the phase plane, we can also investigate local properties such as the directions of maximum stretching and compression, as well as the local vorticity. We easily find that the rate of separation between two nearby points is a maximum in the direction given by

$$
\tan (2 \theta)=\left(1+\rho+\rho_{b}\right) / \gamma,
$$

where $\theta$ defines the angle with the coordinate axis in the $\mu$ space. Thus, in regions of low density, we expect to see lines of mass being stretched with constant positive slope in the phase plane. In the discretized particle picture, this situation corresponds to regions of low density where the particles are not crossing. The above result, Eq. (17), could also have been obtained from this assumption. We will see below that this prediction is accurately born out by simulations.

\section{SIMULATIONS}

An attraction of these one-dimensional gravitational systems is their ease of simulation. In each of the onedimensional Newtonian models considered here (RF, $Q$, frictionless with expansion, and completely autonomous), it is possible to analytically integrate the motion of the individual particles between crossings. Then the temporal evolution of the system can be obtained by following the successive crossings of the individual, adjacent, particle trajectories. In particular, for the $Q$ model, if we let $y_{i}=x_{i+1}-x_{i}$, where we have ordered the particle labels from left to right, then we find that the differential equation for each $y_{i}$ is the same, namely,

$$
\frac{d^{2} y_{i}}{d \tau^{2}}+\frac{1}{\sqrt{6}} \frac{d y_{i}}{d \tau}-y_{i}=-\frac{2 n}{N}
$$

The general solution of the homogeneous version of Eq. (18) is a sum of exponentials. By including the particular solution of the inhomogeneous equation (simply a constant) we obtain a fifth order algebraic equation in $u=\exp (\tau / \sqrt{6})$ for the successive crossings, defined by $y_{j}(\tau)=0$. Hence the name $Q$, or quintic, model. These can be determined numerically in terms of the initial conditions by analytically bounding the roots and employing a numerical root-finding method. (Note that for the RF model a cubic equation is obtained so the crossing times can be found analytically $[35,36,44]$.) A sophisticated, event driven algorithm was designed to execute the simulations. Using the Newton-Raphson method, the algorithm computes all potential crossings of adjacent pairs of particles with double precision accuracy. Since, between crossings, the general solution of the dynamical equations is known analytically, once a crossing time is established, the position and velocity of each particle can be determined with the accuracy of the computer. Two important features of the algorithm are that it only updates the positions and velocities of a pair of particles when they actually cross, and it maintains the correct ordering of each particle's position on the line. In contrast with typical $N$-body simulations, it is not necessary to introduce a discretization length or time step. Using the algorithm we were able to carry out runs for significant cosmological time with large numbers of particles. In particular, it was possible to simulate a system until all of the mass was concentrated in just a few clusters. Depending on the initial conditions, this typically occurred on the order of 15-20 dimensionless time units into the simulation. Since, at this stage, the influence of the boundary conditions could no longer be ignored, there was no point in continuing the runs any further.

Typical numerical simulations were carried out for systems with up to $N=2^{18}$ particles. Initial conditions were chosen by equally spacing the particles on the line and randomly choosing their velocities from a uniform distribution within a fixed interval. For historical reasons we call this a water bag. Other initial conditions, such as normally distributed veloci- 

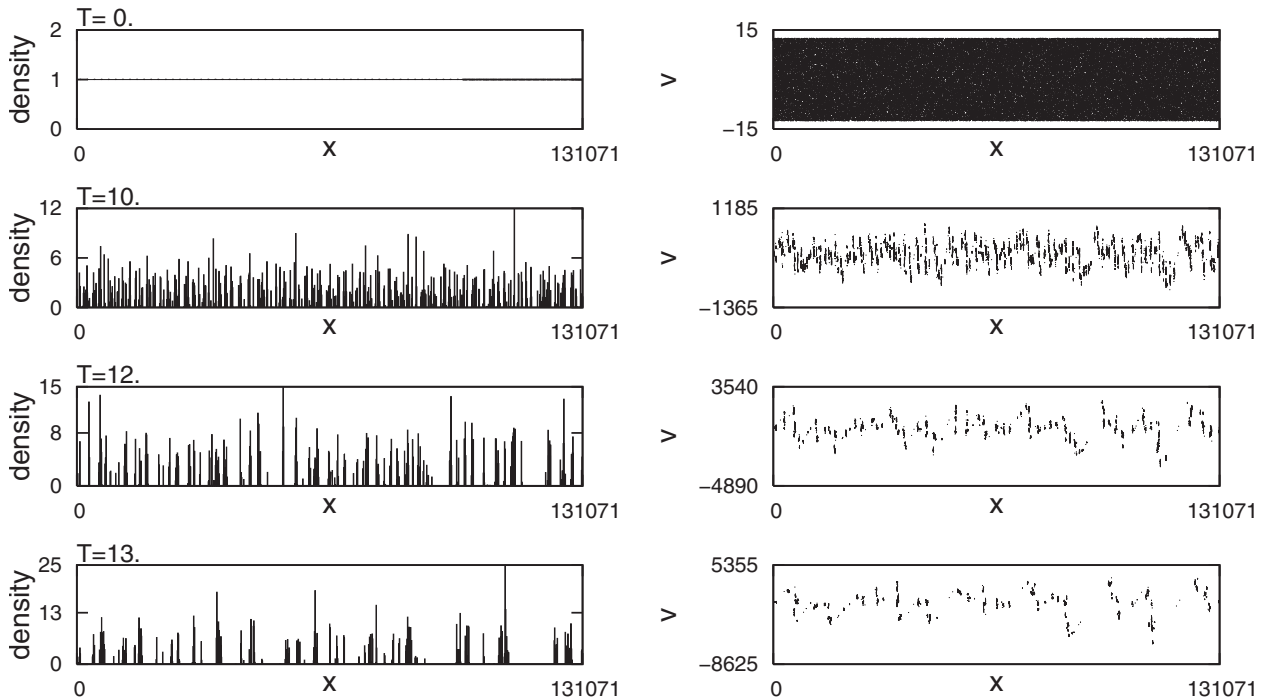

FIG. 1. Evolution in configuration and $\mu$ space for the quintic model with $2^{17}$ particles from $T$ $=0$ to $T=14$. The initial distribution is a water bag with velocities in the range $(-12.5,12.5)$ and a size of about 1700 Jeans' lengths.
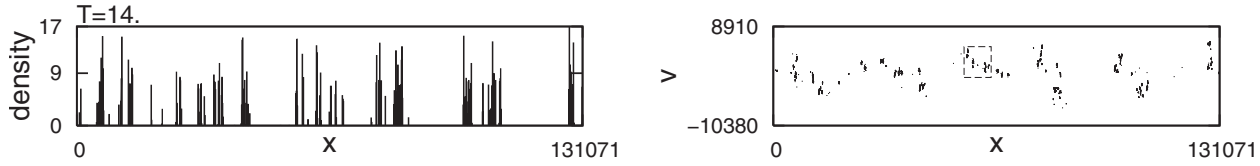

ties, as well as a Brownian motion representation, which more closely imitates a cosmological setting, were also investigated, but only cursorily. For reasons given above, typical simulations were carried forward for approximately fifteen dimensionless time units.

In Fig. 1 we present a visualization of a typical run with $2^{17}$ particles. The system consists of the $Q$ model with an initial water bag distribution in the $\mu$ space. Initially the velocity spread is $(-12.5,12.5)$ in the dimensionless units employed here and the system contains approximately 1700 Jeans' lengths. In the left column we present a histogram of the particles positions at increasing time frames, while on the right we display the corresponding particle locations in $\mu$ space. It is clear from the panels that hierarchical clustering is occurring, i.e., small clusters are joining together to form larger ones, so the clustering mechanism is "bottoms up" [2]. The first clusters are roughly the size of a Jean's length and seem to appear at about $\tau=6$ and there are many while by $\tau=14$ there are on the order of 30 clusters. In the $\mu$ space we observe that between the clusters matter is distributed along linear paths. As time progresses the size of the linear segments increases. The behavior of these underdense regions is governed by the stretching in $\mu$ space predicted by Vlasov theory explained above [see before Eq. (17)]. The slopes of the segments are the same and in quantitative agreement with Eq. (17). Qualitatively similar histories are obtained for the RF model and the model without friction (see below), as well as for the different boundary conditions mentioned above. However, there are some subtle differences. In Fig. 2 we zoom in and show a sequence of two magnified inserts from the mass distribution in $\mu$ space at time $T=14$. The hierarchical structure observed in these models suggests the existence of a fractal geometry, but careful analysis is required to determine if this is correct. Simulations have also been performed where the system size is less than the Jeans' length. The results support the standard stability analysis in that hi-

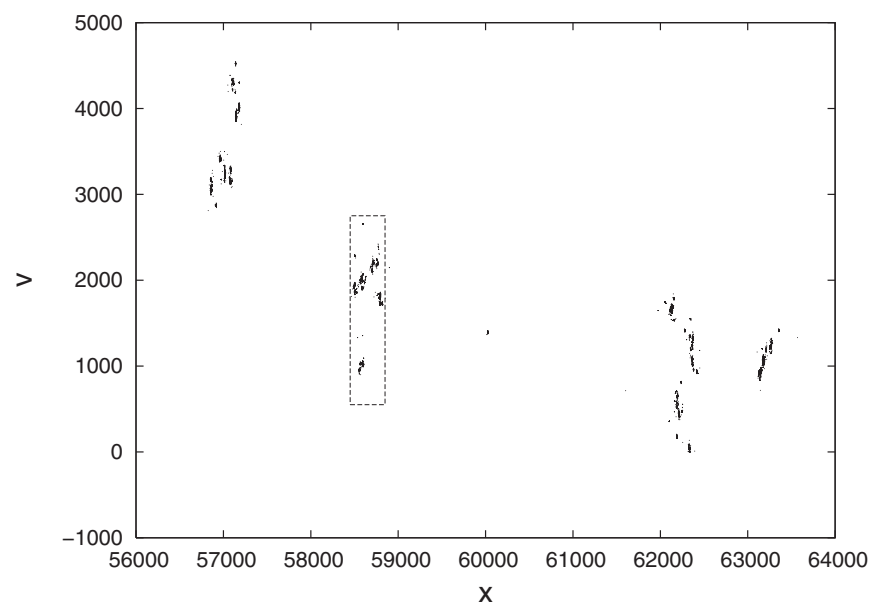

(a)

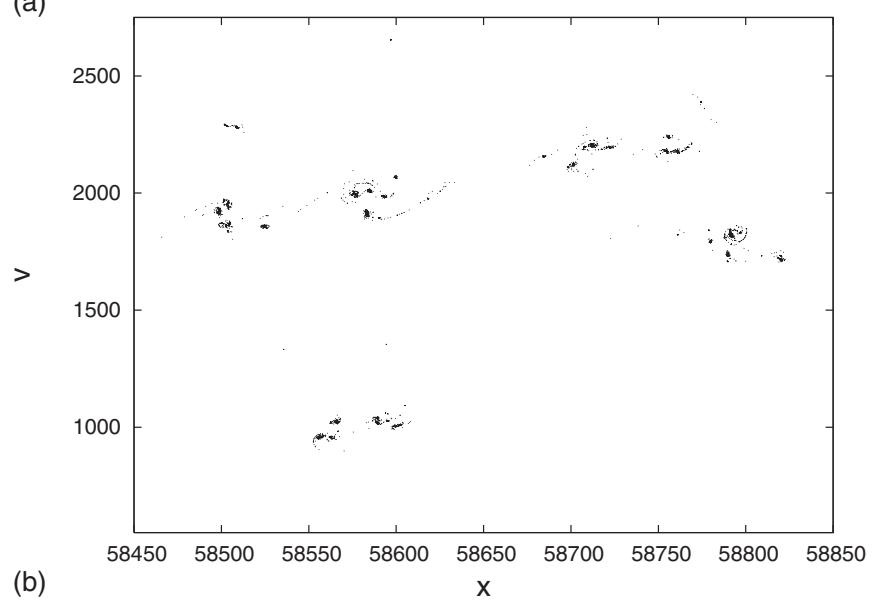

FIG. 2. Consecutive expansions (zooms) on successive small squares selected in the $\mu$ space panels at $T=14$ for the quintic model. They have the appearance of a random fractal, which suggests self-similarity. 


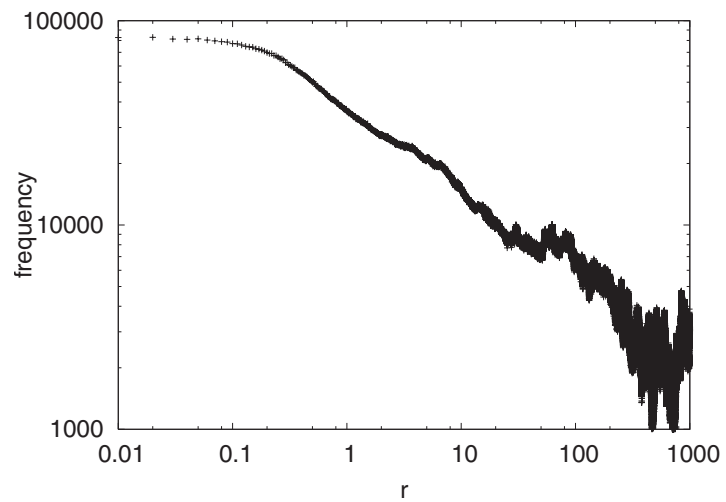

FIG. 3. The correlation function at $T=14$ for the quintic model. It exhibits a scaling region in $r$ from about 0.3 to about 30, a range of about two decades with a scaling exponent $\gamma=0.42$.

erarchical clustering is not observed for these initial conditions.

Historically, power-law behavior in the density-density correlation function has been taken as the most important signature of self-similar behavior of the distribution of galaxy positions [4]. In Fig. 3 we provide a $\log -\log$ plot of the correlation function $C(r)$ at $T=14$ defined by

$$
\begin{aligned}
C(r) & =\langle\delta \rho(x+r) \delta \rho(x)\rangle \\
& \sim \frac{1}{\Delta} \int_{r}^{r+\Delta}\left[\sum_{i, j, i \neq j} \delta\left[r^{\prime}-\left(x_{i}-x_{j}\right)\right]-\frac{N(N-1)}{2 L^{2}}\right] d r^{\prime},
\end{aligned}
$$

where particles $i$ and $j$ are such that $L / 4<x_{i}<3 L / 4$ to avoid boundary effects and $\Delta$ is the bin size. Note the existence of a scaling region from about $0.1<r<30$, a range of about 2.5 decades, where $C \propto r^{-\gamma}$. Note also the appearance of noise at larger scales. This occurs because the correlation function is decreasing while the noise is relatively constant. In a log-log plot, as $r$ is increased, at some point the noise becomes as large as the mean so large fluctuations appear in the data. Expressed differently, in a log-log plot of the correlation function, the apparent noise is effectively magnified with increasing $r$ until it dominates the mean. A straightforward linearization shows that the effective amplitude of the noise in $\ln C$ grows as $\exp (\gamma \ln r)$ while it is still relatively small. In principle it should be possible to reduce the noise by taking an ensemble average of $C(r)$ over many runs, and then computing the slope of $\ln \langle C(r)\rangle$. However, since we already employ $2^{17}$ particles, and the effective noise is growing exponentially with increasing $r$, this may only slightly extend the apparently noise-free region. This is a subject for future study. By computing the slope $\gamma$ of the log-log plot in Fig. 3 we are able to obtain an estimate of the correlation dimension $D_{2}=1-\gamma$ for a one-dimensional system. We find that $\gamma=0.42$ for a scaling region of about two decades in $l$. This suggests that the correlation dimension is approximately 0.6, which is in agreement within the standard numerical error with the multifractal analysis described below in some detail (see Table I).
TABLE I. Generalized dimensions for the quintic and frictionless model.

\begin{tabular}{lccccc}
\hline \hline & \multicolumn{2}{c}{ Quintic model } & & \multicolumn{2}{c}{ Frictionless model } \\
\cline { 2 - 3 } \cline { 5 - 6 }$q$ & $x$ space & $\mu$ space & & $x$ space & $\mu$ space \\
\hline 0 & $0.69 \pm 0.11$ & $0.89 \pm 0.06$ & & $0.89 \pm 0.02$ & $1.14 \pm 0.04$ \\
1 & $0.64 \pm 0.06$ & $0.84 \pm 0.07$ & & $0.88 \pm 0.04$ & $1.17 \pm 0.04$ \\
2 & $0.62 \pm 0.04$ & $0.81 \pm 0.05$ & & $0.85 \pm 0.04$ & $1.13 \pm 0.05$ \\
\hline \hline
\end{tabular}

\section{FRACTAL MEASURES}

It is natural to assume that the apparently self-similar structure that develops in the phase plane (see Figs. 1 and 2) as time evolves has fractal geometry, but we will see that things are not so simple. In their earlier study of the RF model, Rouet and Feix found a box counting dimension for the particle positions in $\mu$ space of about 0.8 for an initial water bag distribution (uniform on a rectangle in the phase plane-see Fig. 1 above) and a fractal dimension of about 0.6 in the configuration space (i.e., of the projection of the set of points in $\mu$ space on the position axis) [36]. As far as we know, Balian and Shaeffer were the first to suggest that the distribution of galaxy positions is consistent with a bifractal geometry [8]. Their idea was that the geometry of the galaxy distribution was different in the clusters and voids and, as a first approximation, this could be represented as a superposition of two independent fractals. Of course, their analysis was restricted solely to galaxy positions. Since the structures which evolve are strongly inhomogeneous, to gain further insight we have carried out a multifractal analysis [47] in both the phase plane and the position coordinate.

The multifractal formalism shares a number of features with thermodynamics. To implement it we partitioned each space, configuration space and $\mu$ space, into cells of length $l$. At each time of observation in the simulation, a measure $\mu_{i}=N_{i}(t) / N$ was assigned to cell $i$, where $N_{i}(t)$ is the population of cell $i$ at time $t$ and $N$ is the total number of particles in the simulation. The generalized dimension of order $q$ is defined by [47]

$$
D_{q}=\frac{1}{q-1} \lim _{l \rightarrow 0} \frac{\ln C_{q}}{\ln l}, \quad C_{q}=\Sigma \mu_{i}^{q},
$$

where $C_{q}(l)$ is the effective partition function [9], $D_{0}$ is the box counting dimension, $D_{1}$, obtained by taking the limit $q$ $\rightarrow 1$, is the information dimension, and $D_{2}$ is the correlation dimension $[9,47]$. As $q$ increases above 0 , the $D_{q}$ provide information on the geometry of cells with higher population.

In practice, it is not possible to take the limit $l \rightarrow 0$ with a finite sample. Instead, one looks for a scaling relation over a substantial range of $\ln l$ with the expectation that a linear relation between $\ln C_{q}$ and $\ln l$ occurs, suggesting power-law dependence of $C_{q}$ on $l$. Then, in the most favorable case, the slope of the linear region should provide the correct power and, after dividing by $q-1$, the generalized dimension $D_{q}$. As a rule, or guide, if scaling can be found either from observation or computation over three decades of $l$, then we typically infer that there is good evidence of fractal structure [7]. Also 

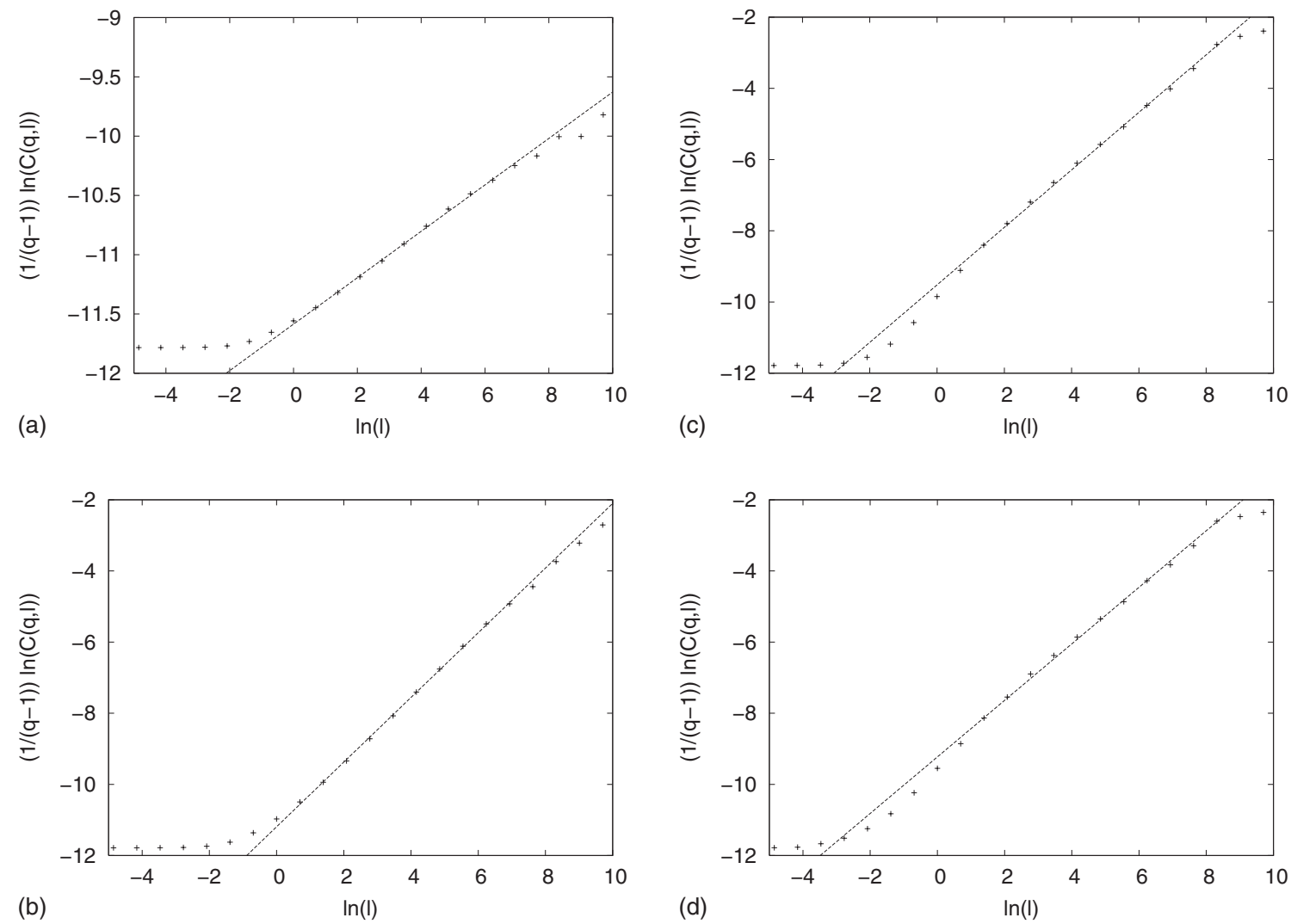

FIG. 4. Scaling behavior in $\mu$ space. Plots of $\frac{1}{q-1} \ln C_{q}$ versus $\ln l$ are provided for four values of $q$ : (a) $q=-5$, (b) $q=0$, (c) $q=5$, and (d) $q=10$.

of interest is the global scaling index $\tau_{q}$, where $C_{q} \sim l^{\tau_{q}}$ for small $l$. It can be shown that $\tau_{q}$ and $D_{q}$ are related to each other through a Legendre transformation by $D_{q}(1-q)=\tau_{q}$ for $q \neq 1$ [9]. Here we present the results of our fractal analysis of the particle positions on the line (position only) and the plane (position, velocity, or $\mu$ space).

If it exists, a scaling range of $l$ is defined as the interval on which plots of $\ln C_{q}$ versus $\ln l$ are linear. Of course, for the special case of $q=1$ we plot $-\Sigma \mu_{i} \ln \mu_{i}$ vs $\ln l$ to obtain the information dimension [9]. If a scaling range can be found, $D_{q}$ is obtained by taking the appropriate derivative. It is well established by proof and example that, for a normal, homogeneous fractal, all of the generalized dimensions are equal, while for an inhomogeneous fractal, e.g., the Henon attractor, $D_{q+1} \leqslant D_{q}$ [47]. In the limit of small $l$, the partition function $C_{q}(l)$ can also be decomposed into a sum of contributions from regions of the inhomogeneous fractal sharing the same pointwise dimension $\alpha$,

$$
C_{q}(l)=\int d \alpha l^{\alpha q} \rho(\alpha) \exp [-f(\alpha)],
$$

where $f(\alpha)$ is the fractal dimension of its support $[9,47,48]$. Then if, for a range of $q$, a single region is dominant, we find a simple relation between the global index $\tau_{q}$ and $\alpha$,

$$
\tau_{q}=\alpha q-f(\alpha)
$$

and a corresponding linear relation between $\ln C_{q}$ and $q$.
Recall that a Euclidean metric is imposed on the $\mu$ space. Because, in our units, $\omega_{j}=1$, we divide it into cells such that $\Delta x=\Delta v$. Then, as $L$ is large $(L \approx 2000)$, so is the extent of the partition in the velocity space. On this scale the initial distribution of particles appears as a line so that the initial dimension is also about unity. In fact, initially the velocity of the particles is a perturbation. It is not large enough to allow particles to cross the entire system in a unit of time. After a while the granularity of the system destroys the approximate symmetry of the initial $\mu$ space distribution. Breaking the symmetry leads to the short-time dissipative mixing that results in the separation of the system into clusters. Although the embedding dimension is different, the behavior of the distribution of points in configuration space is similar. The initial dimension is nearly one until clustering commences. At this time the dimensions in $\mu$ space and configuration space separate.

As time progresses, however, for the initial conditions discussed above, for $q>0$ typically two independent scaling regimes develop. Of course this is in addition to the trivial scaling regions obtained for very small $l$, corresponding to isolated points, and to large $l$ on the order of the system size, for which the matter distribution looks smooth. The observed size of each scaling range depended on both the elapsed time into the simulation and the value of $q$. While the length of each scaling regime varied with both $q$ and time, in some instances it was possible to find good scaling over up to four decades in $l$.

In Fig. 4 we provide plots of $\frac{1}{q-1} \ln C_{q}$ versus $\ln l$ in $\mu$ 

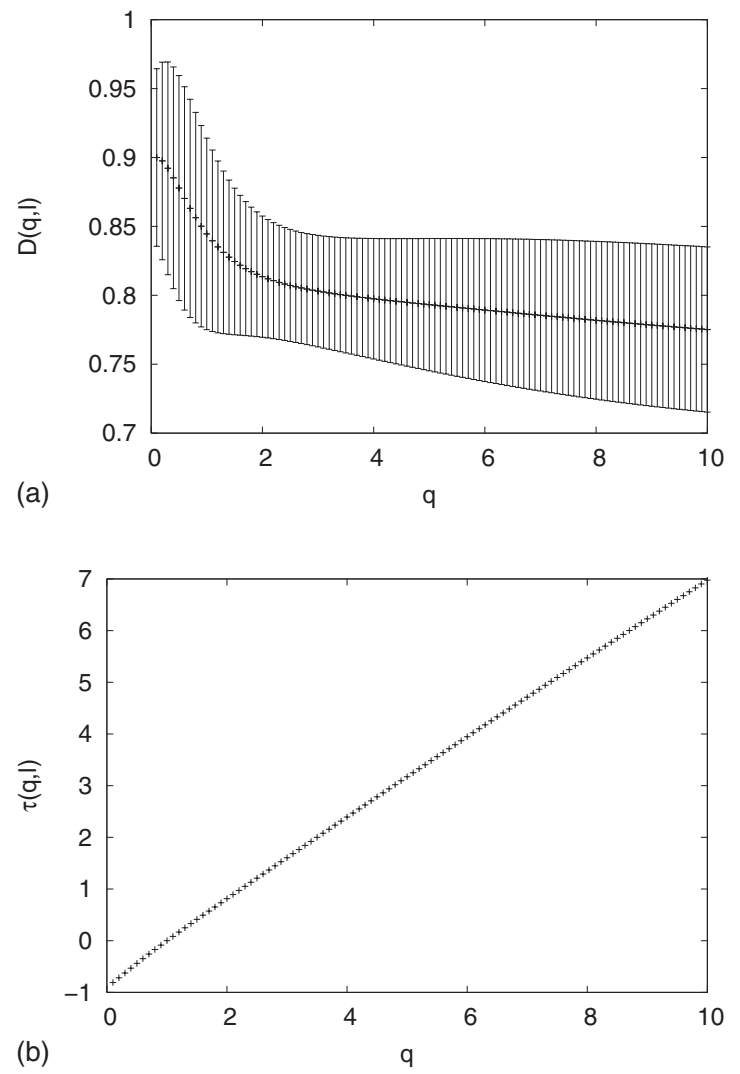

FIG. 5. Generalized dimension $D_{q}$ vs $q$ [panel (a)] and global scaling index $\tau_{q}$ vs $q$ [panel (b)] in $\mu$ space for the quintic model at $T=14$ with $q>0$.

space for four different values of $q$ covering most of the range we investigated, $(-5<q<10)$. To guarantee that the fractal structure was fully developed, we chose $T=14$ for the time of observation and the initial conditions are those given above. For $q=-5$ and $q=0$ we clearly observe a single, large, scaling range where $0.5<\ln l<8$, corresponding to about three decades in $l$. In the remaining panels [(c), (d)], where we increase $q$ to 5 , and then 10 , we see a dramatic change. The large scaling range has split into two smaller regions separated at about $l=1.5$, and the slope of the region with larger $l$ has decreased compared with the scaling region on its left $(-1.5<\ln l<1.5)$. The scaling range with larger $l$, approximately $1.5<\ln l<8.5$, corresponds to just over three decades in $l$. Note that in panels (c) and (d) of Fig. 4, the scaling range with larger $l$ is more robust. The straight (dashed) lines that appear in the data are best fits obtained from linear regression for the larger scaling region. Their slopes were used to compute the generalized dimensions.

Now that we have identified the important scales, we are able to compute the generalized dimension $D_{q}$, and the global index $\tau_{q}$. In Fig. 5(a) we plot $D_{q}$ vs $q$ calculated in the $\mu$ space for the quintic model at $T=14$ for $q>0$ for the case of the larger scaling range. As expected, it is a decreasing function and clearly demonstrates multifractal behavior. Although $\mu$ space is two dimensional, $D_{0}$ is about 0.9 . While $D_{0}$ $>D_{1}$ is typical for multifractals (information dimension covers the important region), the fact that $D_{q}$ is strictly decreasing suggests greater "fractality" from increasingly overdense regions, i.e., the system is strongly inhomogeneous. In Fig. 5(b) we plot $\tau_{q}$ vs $q$ in $\mu$ space. Two linear regions $(0<q$ $<1$ and $1<q<10$ ) can be distinguished suggesting bifractal geometry, i.e., a superposition of two fractals with unique values of $\alpha$ and $f(\alpha)$.

We have seen that the one-dimensional gravitational system reveals fractal geometry in the higher-dimensional $\mu$ space (frequently referred to as phase space in the astronomical literature). Historically, self-similar behavior was first inferred in gravitational systems by studying the distribution of galaxy locations, i.e., in the system's configuration space, and searching for power-law behavior in the correlation function (see above) [8]. Following this approach, we project the $\mu$ space distribution on the (position) line to study the geometry of the configuration space. In Figs. 6(a)-6(d), we provide plots of $1 /(q-1) \ln C_{q}$ vs $\ln l$ in $x$ (configuration) space for the quintic model prepared as above at $T=14$. In common with the $\mu$ space distribution, for $q=-5$ and $q=0$, there is a large scaling region $(-3<\ln l<3$ for $q=-5$ and $-1<\ln l<4$ for $q=0)$. However, the hint of a second scaling region also appears and becomes more prominent for $q>0$. At $q=5$ (c) and $q=10$ (d) the existence of two scaling ranges becomes very apparent, $-4<\ln l<0$ and $0<\ln l<8$ for $q$ $=5$ (about 3.3 decades), $-3<\ln l<1$ and $1<\ln l<8$ for $q$ $=10$ (about three decades). Note that the scaling ranges are similar for both the $\mu$ and configuration space, but they are not identical. The straight lines that appear in the plots are best fits to the data over the larger interval.

In Fig. 7 we illustrate the behavior of the generalized dimension $D_{q}$ and global index $\tau_{q}$ for the configuration space of the quintic model at $T=14$. Although now the embedding dimension is $d=1, D_{0}$ is about 0.7 so the distribution is definitely fractal. As anticipated from the $\mu$ space distribution, $D_{q}$ is a decreasing function of $q$ so it is also inhomogeneous and multifractal. In Fig. 7(b) we plot $\tau_{q}$ vs $q$ in configuration space, for the same system. In contrast with the $\mu$ space index, here we observe a nearly linear function with slight convexity (decreasing derivative with respect to $q$ ). Perhaps this results from the superposition of three linear regions with distinct $\alpha$ and $f(\alpha)$, say with $0<q<1.5 ; 1.5<q<7$; $7<q<10$, but this cannot be inferred from the plot without further analysis.

For completeness, in Fig. 8 we examine the behavior of $D_{q}$ and $\tau_{q}$ for $q<0$. In general, negative $q$ is important for revealing the geometry of low density regions (voids). We see that $D_{q}$ has a very unphysical appearance - it is increasing. The source of the problem can be determined by examining the behavior of $\tau_{q}$. It is nearly constant over the range $-10<q<0$ with a value of approximately $\tau_{q} \simeq-1$, implying that the underdense regions are dominated by a single local dimension.

Part of our goal is to compare how fractal geometry arises in a family of related models. So far we have presented results for the quintic, or $Q$, model. However, we have also carried out similar studies of the Rouet-Feix (RF) model, the model without friction [which can be obtained from either of the former by nullifying the first derivative contribution in Eq. (13)], and simply an isolated system without friction or background. The latter is a purely Newtonian model without a cosmological connection. In Table I we list the important 

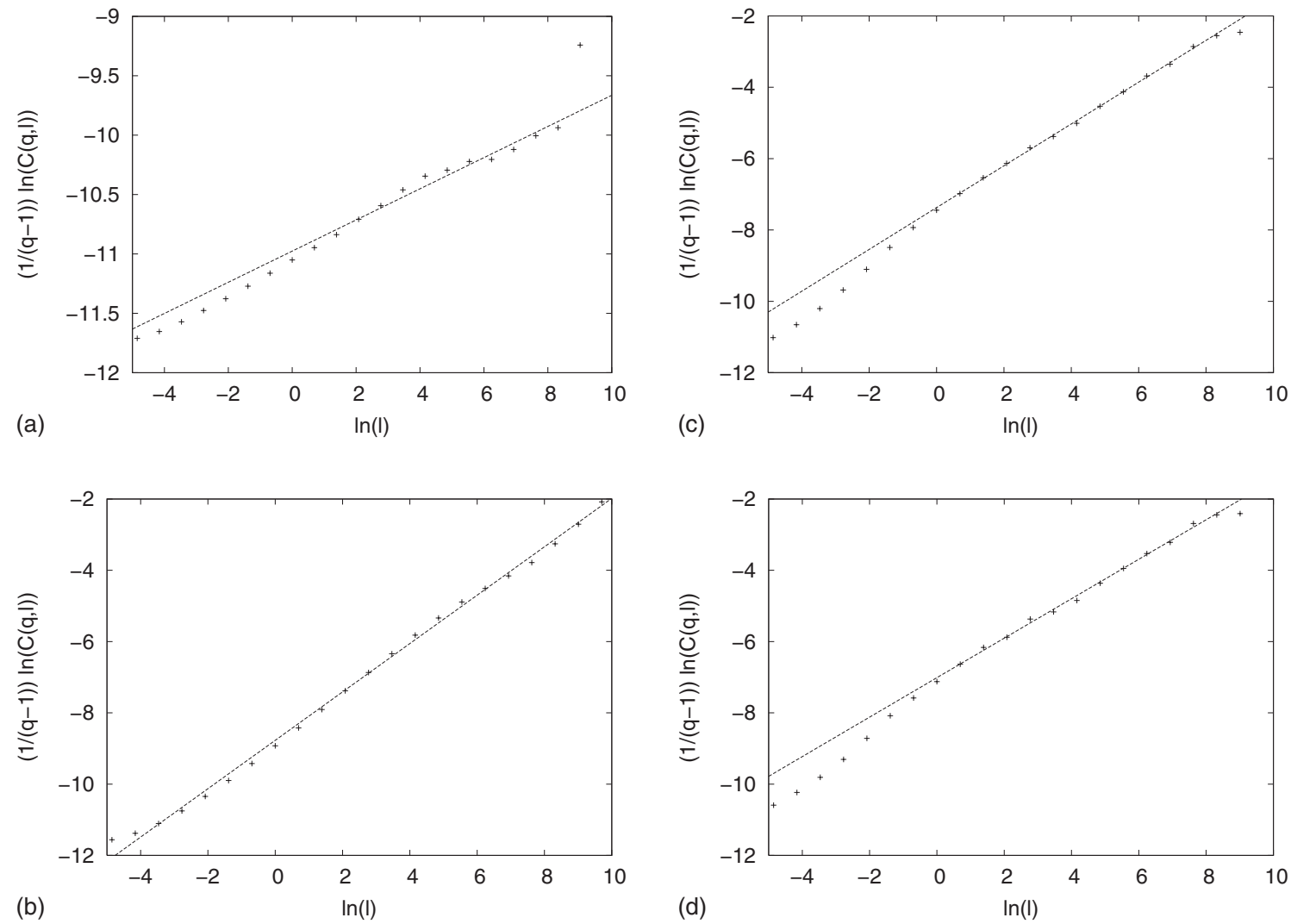

FIG. 6. Plots of $1 /(q-1) \ln C_{q}$ vs $\ln l$ in configuration $(x)$ space for the quintic model prepared as above at $T=14$ are provided for four values of $q$ : (a) $q=-5$, (b) $q=0$, (c) $q=5$, and (d) $q=10$.

characteristic dimensions and exponents for the the quintic model and the model without friction for comparison. While there are similarities in the fractal structure of each of these models, they are not the same. For example, in the quintic model the generalized fractal dimension $D_{q}$ is consistently smaller in each manifold than the corresponding dimension in the model without friction. It is noteworthy that the boxcounting dimension in the configuration space of the quintic model is 0.69 compared with 0.90 for the model without friction. Moreover, $D_{q}$ is decreasing more rapidly in the quintic model demonstrating stronger inhomogeneity.

The differences in dynamics between the systems can be seen clearly in Fig. 9, where we show the evolution in configuration and $\mu$ space of the system without friction. In common with the simulations of the quintic model presented above, the initial conditions are a water bag with velocities sampled independently and confined to $(-12.5,12.5)$ in the dimensionless units defined earlier. As before, there are $2^{17}$ particles and the simulation spans 14 units of the scaled time. The initial size encompasses about 1700 Jeans lengths. Compared with the quintic model, note that here larger clusters form earlier and have a qualitatively different shape. Due to the absence of "friction" in the dynamics, the velocity spread is larger and there are fewer clusters at each epoch. At $T$ $=12$ and $T=13$ the linear structure of the underdense regions (voids) in $\mu$ space connecting the clusters is apparent. Note that they all share a common slope that is due to stretching in the phase plane. However, Eq. (17) is no longer adequate to provide the line slope. In Fig. 10 we examine the consecutive expansions (zooms) of the large cluster on the right hand side of the $\mu$ space distribution at $T=14$. Once again there is qualitative evidence of stochastic self-similarity [9] that requires analysis for confirmation. To save space, here we do not reproduce the plots of $\frac{1}{q-1} \ln \left(C_{q}\right)$ vs $\ln l$ in each space. Qualitatively they are similar to the quintic case, but the scaling ranges are less robust.

The behavior of $D_{q}$ and $\tau_{q}$ are similar to that found for the quintic model. However, because the scaling ranges are smaller, there is more noise. For example, in Figs. 11(a) and 11(b), we plot $D_{q}$ and $\tau_{q}$ vs $q$ in configuration space for the model without friction. Note the similarities with Fig. 6 for the quintic model. The slope of $\tau_{q}$ gradually decreases with increasing $q$. There appears to be three linear segments, from $(0,2),(2,7)$, and $(7,10)$ suggesting contributions from three possible fractal scaling regions.

\section{DISCUSSION OF RESULTS}

As mentioned earlier, for a regular multifractal the generalized dimension $D_{q}$ is a decreasing function of its argument $[9,48]$. Therefore, for $q<0$, it would be incorrect to interpret the simulation results as true generalized dimensions. There must be an alternative explanation for the behavior we observe. The picture for positive $q$ is rather different. The two scaling regions give completely different results. Although one would suspect from the definition of the generalized dimension that the scaling region with smaller $l$ would give the correct one, this is hard to accept since typical plots of the 

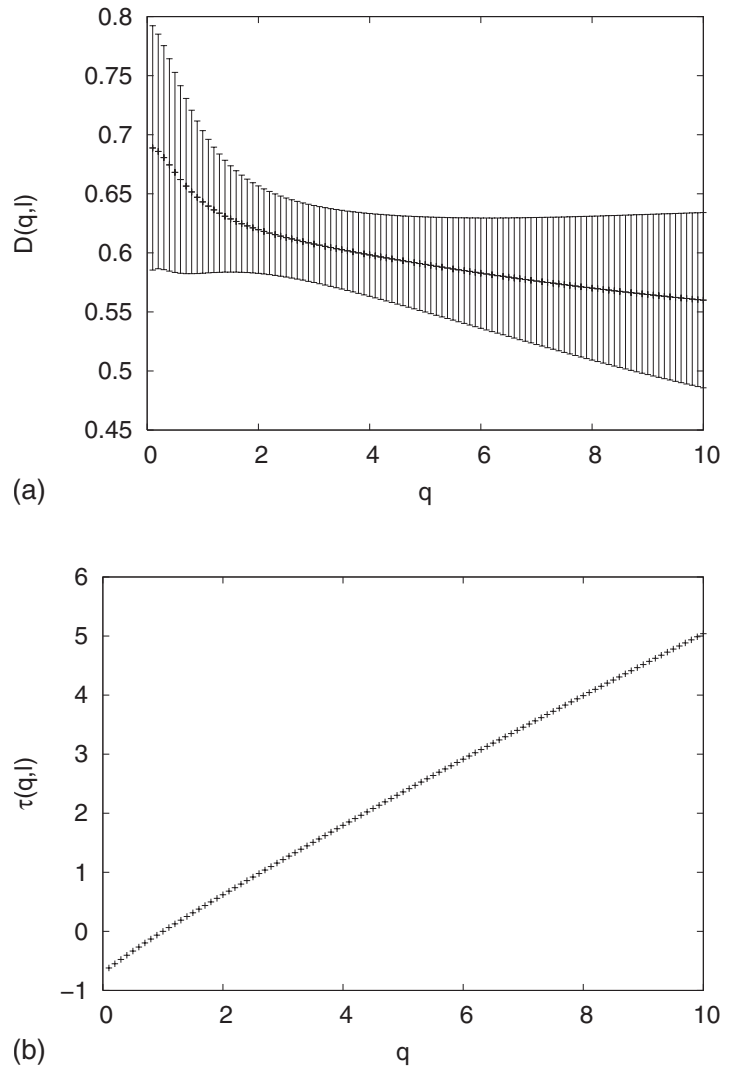

FIG. 7. Generalized dimension $D_{q}$ vs $q$ [panel (a)] and global scaling index $\tau_{q}$ vs $q$ [panel (b)] in configuration $(x)$ space for the quintic model at $T=14$ with $q>0$.

function $D_{q}$ are still increasing until about $q=2$ (not shown) for this range. On the other hand, the second, larger, scaling region manifests a well behaved decreasing function, which appears to approach a constant value of $D_{q} \cong 0.63$ for $q$ $\lesssim 10$.

It is significant that we have observed similar behavior with a well characterized, textbook fractal that is discussed in numerous sources [48]. As a test of our computational approach we simulated the multiplicative binomial process. For this multifractal $\tau_{q}$ and $D_{q}$ are known precisely [48]. When we carried out the fractal analysis using the methods described above, we also found two scaling regions. What is most striking is that the scaling region with larger values of $l$ yielded a $\tau_{q}$ (and therefore $D_{q}$ ), which agreed to within numerical error with the theoretical prediction. This seemingly anomalous behavior has been manifested in other simulations of fractal sets. The formation of the smaller scaling range has been attributed to the presence of noise in the data. The existence of a second scaling range has been rigorously shown to arise when Gaussian noise is added to a standard fractal [49]. In the simulation of the multiplicative binomial process, the source of the noise is the random location of the data points within the smallest bins. At this time the source of the apparent noise in the one-dimensional gravitational simulations is not precisely known. While it may arise simply from numerical considerations, there are alternative possible explanations. For example, noise may arise from subJeans length fluctuations in the initial data, or from other
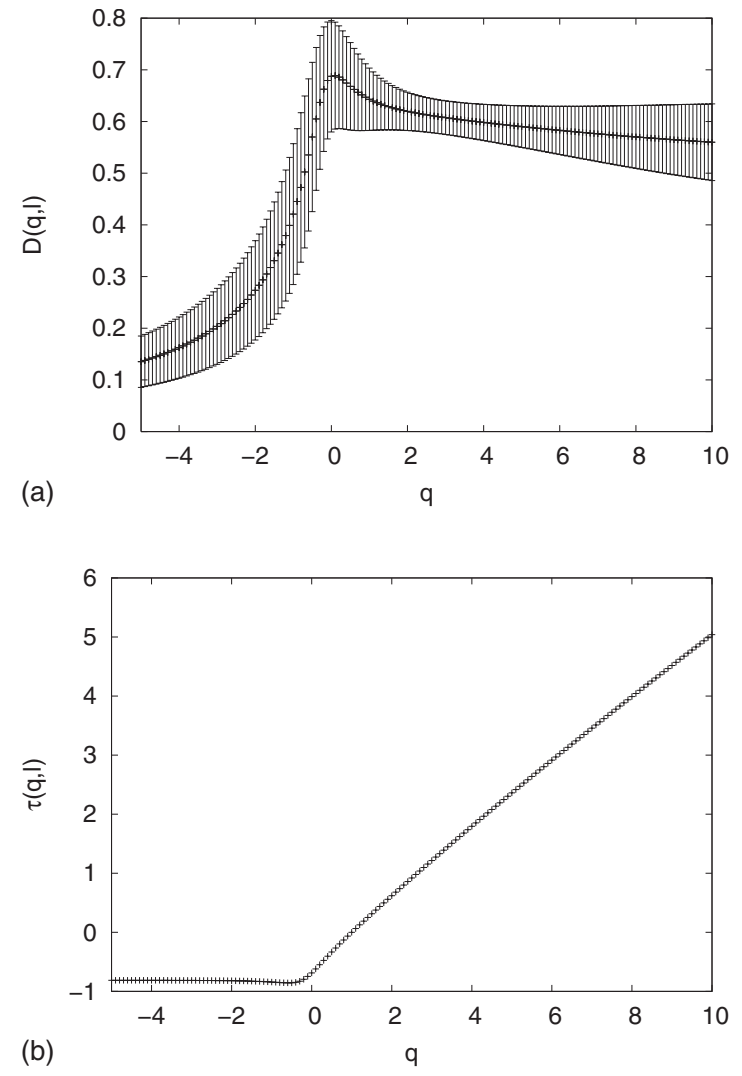

FIG. 8. Generalized dimension $D_{q}$ vs $q$ [panel (a)] and global scaling index $\tau_{q}$ vs $q$ [panel (b)] in configuration $(x)$ space for the quintic model at $T=14$ with $q<0$.

small scale features of the initial state. In addition to the box-counting approach, another method based on the correlation function formalism that employs the pointwise dimension can also be used to investigate the fractal properties of the system [9]. It was also used in our investigation and gives similar results to the box-counting method reported here.

For $q>0$ we appear to be seeing a similar phenomena to the multiplicative binomial process or the systems with injected noise [49]. Then how do we explain the surprising and counterintuitive results for $q<0$ ? Since for negative $q$ we obtain a nearly constant value for $\tau_{q}$ from each scaling region, it seems safe to assume that a region of the data characterized by a simple fractal behavior has the dominant influence. Moreover, since it only involves $q<0$, it represents the regions of low density, i.e., the voids. Referring to the multifractal formalism, we expect Eq. (22) to apply in a region where a single structure dominates. Then the computations show that for $q<0$ we must have $\alpha=0$ and $f(\alpha) \cong 0.9$. This suggests that the results for negative $q$ are dominated by regions of such low density that widely separated, "isolated" particles are responsible for the spurious behavior of $D_{q}$. At this time this is simply a conjecture that needs to be investigated with further computation.

Finally, let us reconsider the plot of $\tau_{q}$ versus $q$ [Fig. 5(b)] for the larger scaling region with $q>0$. As we mentioned earlier, for $0<q<1.5$ and for $2<q<10$ the curve appears linear with different slopes in each region. This may be the 

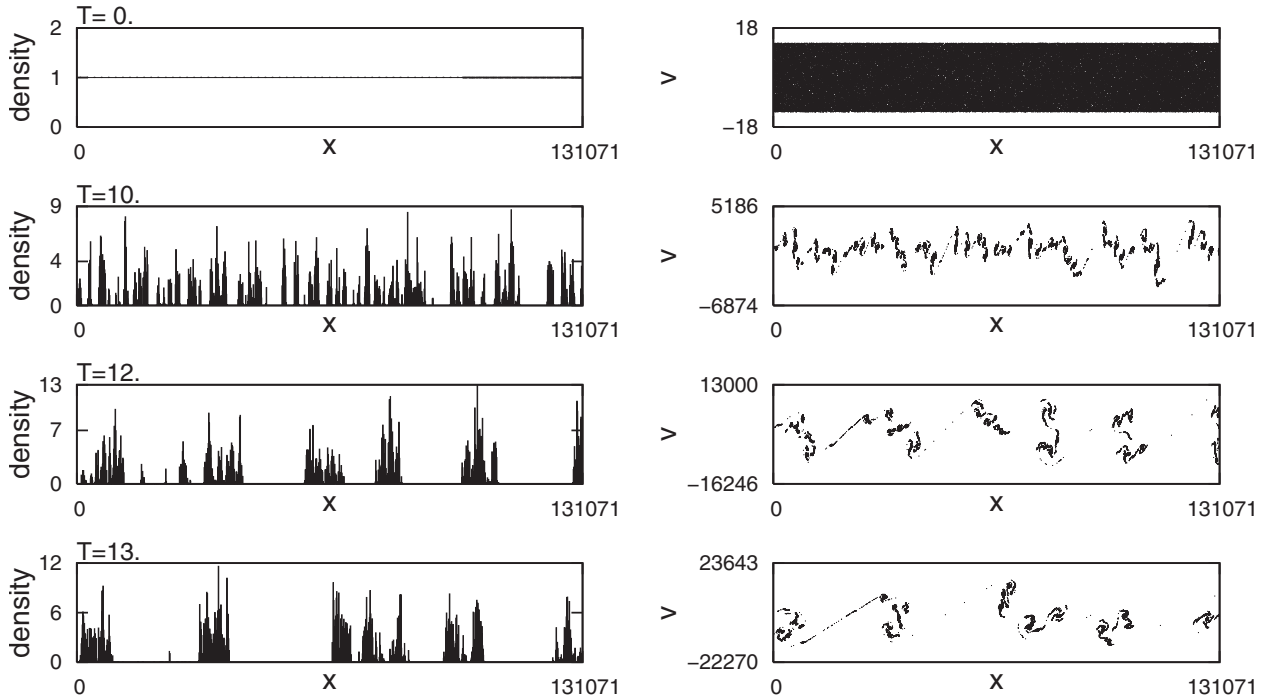

FIG. 9. Evolution in configuration and $\mu$ space for the model without friction with $2^{17}$ particles from $T=0$ to $T=14$. The initial distribution is a water bag with velocities in the range $(-12.5$, 12.5) in the dimensionless units employed here and a size of about 1700 Jeans' lengths.
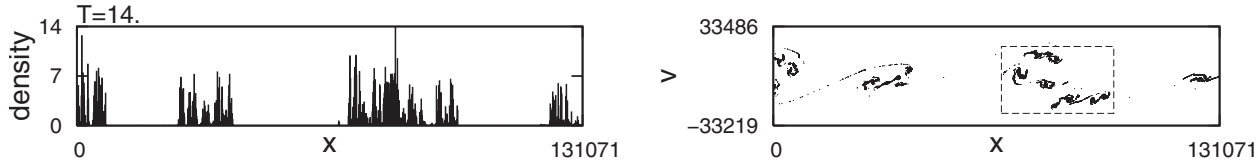

manifestation of bifractality first discussed by Balian and Shaeffer for galaxy positions. The first interval may represent the true fractal structure of the underdense regions, while the dense clusters are dominant for the larger $q$ values. Bifractal behavior is not unique to gravitational systems. It occurs in a number of well studied model systems, for example, as a superposition of two Cantor sets [50], or from the truncation of Levy flights $[51,52]$.

\section{CONCLUSIONS}

Clearly one-dimensional models develop hierarchical structure and manifest robust scaling behavior over particular length and time scales. In addition, for a number of reasons, they have a computational advantage over higherdimensional models. First, it is possible to study the evolution of large systems with on the order of $2^{17}$ particles per dimension. Second, in contrast with three-dimensional $N$-body simulations, the evolution can be followed for long times without compromising the dynamics. In particular, the force is always represented with the accuracy of the computer-there is no softening or imposed grid. Third, since the motion can be determined analytically between particle crossings, it is not necessary to discretize either space or time. As a consequence, it is possible to investigate the prospect of fractal geometry with confidence.

In common with $(3+1)$-dimensional cosmologies, with the inclusion of the Hubble expansion and the transformation to the comoving frame, both the $(1+1)$-dimensional quintic and RF [44] models reveal the formation of dense clusters and voids. They also show evidence for bifractal geometry. This may be a consequence of dynamical instability that results in the separation of the system into regions of high and low density. An interesting observation is that the lower bound of the length scale that supports the trivial space dimension of unity in the configuration space grows with time.
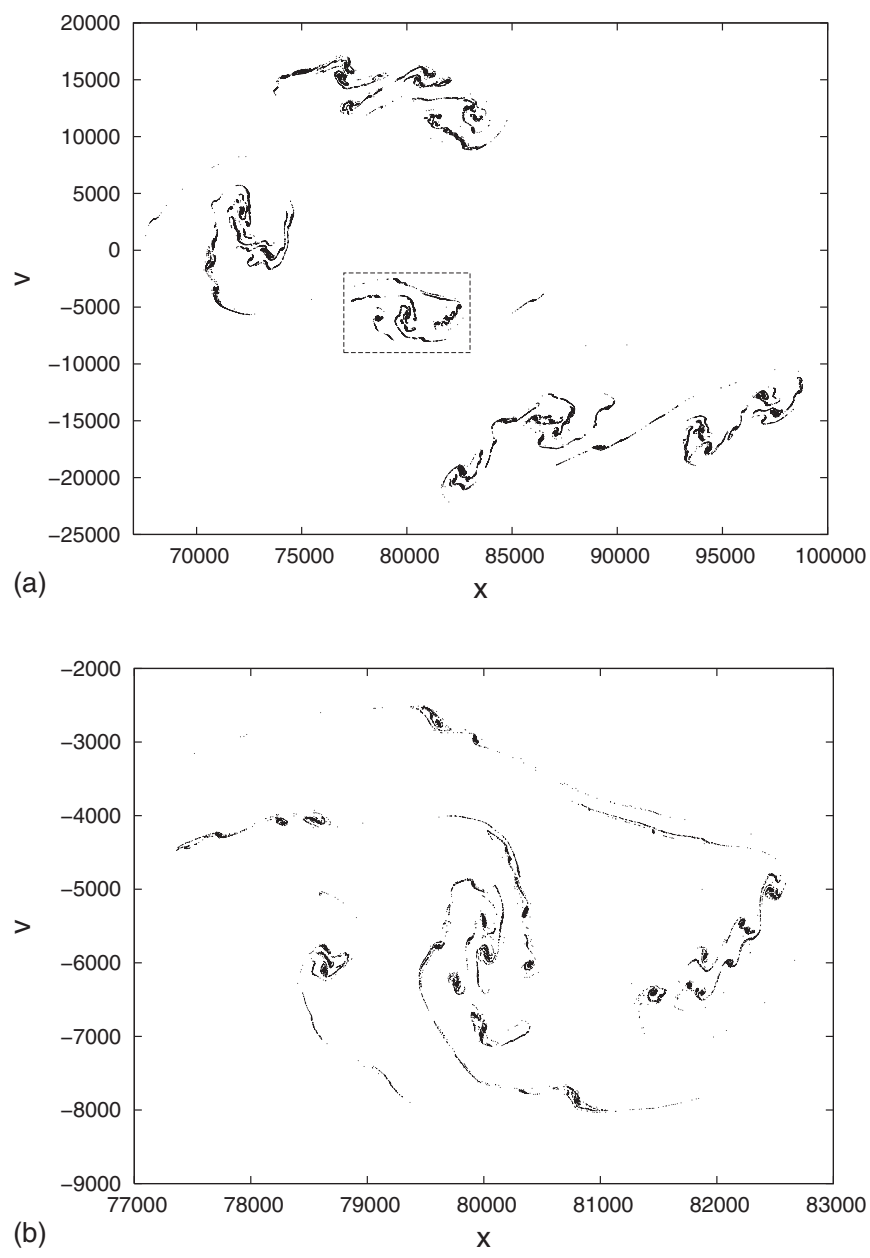

FIG. 10. Consecutive expansions (zooms) of successive small squares (square within a square) selected in the $\mu$ space panels at $T=14$. They also have the appearance of a random fractal, which suggests self-similarity. 

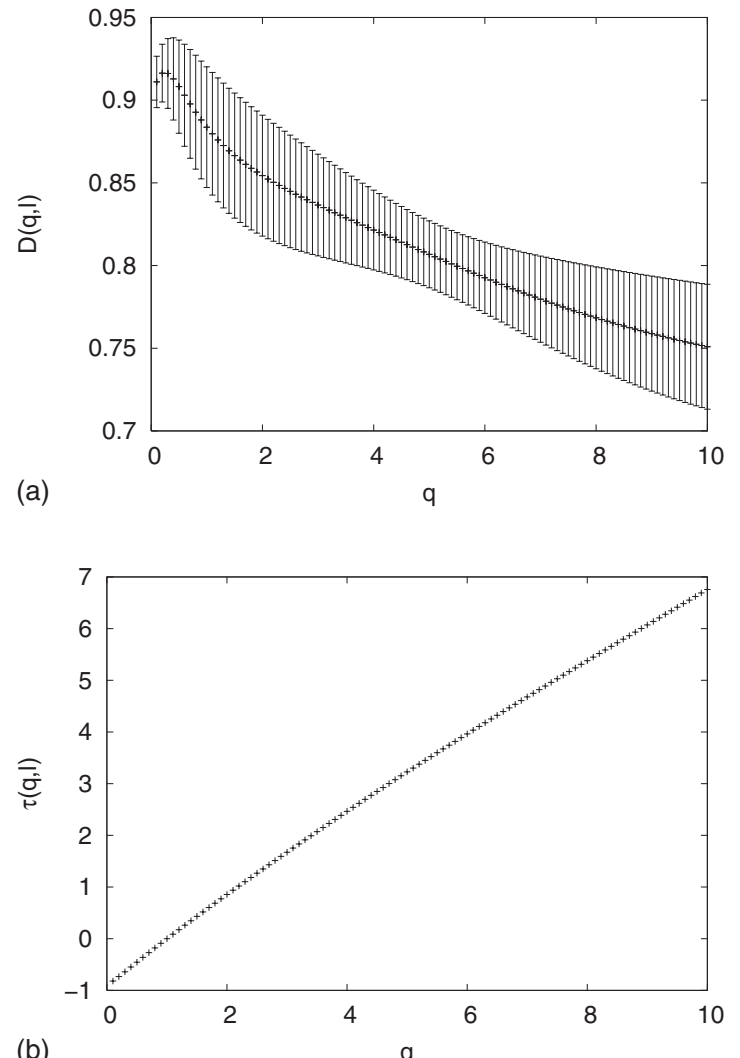

(b)

q

FIG. 11. Generalized dimension $D_{q}$ vs $q$ [panel (a)] and global scaling index $\tau_{q}$ vs $q$ [panel (b)] in configuration (x) space for the quintic model at $T=14$ with $q>0$.

To the extent that similar behavior occurs in the $(3+1)$-dimensional universe, this lends support for the standard cosmological model on sufficiently large scales. It also suggests that the scale size for homogeneity will grow with time. Eventually this may be testable with observation.

We have seen that the system shows evidence of two nontrivial scaling regions. The type of anomalous behavior of $D_{q}$ and $\tau_{q}$ in the scaling region with a finer partition was also found in the standard multiplicative binomial process with a similar sample size. Even with the large sample size employed here, this would suggest that it is a finite size effect. In that respect it will be interesting to perform simulations dealing directly with the distribution function given by the Vlasov equation. Then the low density region may be well described. This may also be true for the region of negative $q$ where a fractal analysis forces us to conclude that the pointwise dimension vanishes. Computations with different initial conditions appear to reveal similar behavior, but this work is only in the preliminary stages. Future work will include the investigation of the influence of correlation in the initial conditions, the consideration of initial conditions of the type employed in current cosmological $N$-body simulations [53], and the study of the fractal geometry of the underdence and overdense regions. We plan to compare our findings with three-dimensional studies of the distribution of voids and halos [54].

In addition to these important features, the onedimensional simulations unequivocally demonstrate that the essential structure formation is taking place in the $\mu$ space. Thus the apparent fractal geometry that we observe in configuration space is simply a shadow (projection) of the higher-dimensional features. The development of structure in $\mu$ space and its apparent fractal geometry may be the most significant result of these studies. Since evolution in the sixdimensional $\mu$ space of the observable universe is beyond our current capability, the study of lower-dimensional models provides a useful guide for understanding the important features of the higher-dimensional evolution. For example, we have shown analytically that dynamical "stretching" of the $\mu$ space geometry is responsible for the formation of underdense regions (voids) and, consequently, the concentration of mass in regions of decreasing area. We are currently extending this analysis to the study of structure formation in the more realistic $(3+1)$-dimensional manifold.

\section{ACKNOWLEDGMENTS}

A sabbatical leave from Texas Christian University and the hospitality and support from the Université d'Orléans is gratefully acknowledged by B.N.M.
[1] G. de Vaucouleurs, Science 167, 1203 (1970).

[2] P. J. E. Peebles, Principles of Physical Cosmology (Princeton University Press, Princeton, NJ, 1993).

[3] J.-C. Pecker and J. Narlikar, Current Issues in Cosmology (Cambridge University Press, Cambridge, UK, 2006), Vol. 167.

[4] B. Jones, V. Martinez, E. Saar, and V. Trimble, Rev. Mod. Phys. 76, 1211 (2004).

[5] B. Mandelbrot, The Fractal Geometry of Nature (Freeman, San Francisco, 1982).

[6] F. S. Labini, M. Montuori, and L. Pietronero, Phys. Rep. 293, 61 (1998).

[7] J. L. McCauley, Physica A 309, 183 (2002).

[8] R. Balian and R. Schaeffer, Astron. Astrophys. 226, 373 (1989).
[9] E. Ott, Chaos in Dynamical Systems (Cambridge, Cambridge, UK, 2002).

[10] J. Hwang and H. Noh, Mon. Not. R. Astron. Soc. 367, 1515 (2006).

[11] H. de Vega and N. Sanchez, Phys. Lett. B 490, 180 (2000).

[12] P. V. Grujic, Astrophys. Space Sci. 295, 363 (2005).

[13] J. Bagla, Curr. Sci. 88, 1088 (2005).

[14] E. Bertschinger, Annu. Rev. Astron. Astrophys. 36, 599 (1998).

[15] T. P. Weissert, The Genesis of Simulation in Dynamics (Springer-Verlag, New York, 1997).

[16] L. Cohen and M. Lecar, Bull. Astron. 3, 213 (1968).

[17] F. Hohl, Ph.D. thesis, College of William and Mary, 1967 (unpublished).

[18] H. Wright, B. Miller, and W. Stein, Astrophys. Space Sci. 84, 
421 (1982).

[19] K. R. Yawn and B. N. Miller, Phys. Rev. E 56, 2429 (1997).

[20] K. R. Yawn and B. N. Miller, Phys. Rev. Lett. 79, 3561 (1997).

[21] H. A. Posh, H. Narnhofer, and W. Thirring, Physica A 194, 482 (1993)

[22] L. Milanovic, H. A. Posch, and W. Thirring, Phys. Rev. E 57, 2763 (1998).

[23] L. Milanovic, H. A. Posch, and W. Thirring, J. Stat. Phys. 124, 843 (2006).

[24] J.-L. Rouet and M. R. Feix, Phys. Rev. E 59, 73 (1999).

[25] B. Miller, K. Yawn, and B. Maier, Phys. Lett. A 346, 92 (2005).

[26] V. P. Youngkins and B. N. Miller, Phys. Rev. E 56, R4963 (1997).

[27] P. Youngkins and B. Miller, Phys. Rev. E 62, 4583 (2000).

[28] P. J. Klinko and B. N. Miller, Phys. Rev. E 62, 5783 (2000).

[29] P. Klinko, B. N. Miller, and I. Prokhorenkov, Phys. Rev. E 63, 066131 (2001).

[30] P. Klinko and B. N. Miller, Phys. Rev. E 65, 056127 (2002).

[31] P. Klinko and B. N. Miller, Phys. Rev. Lett. 92, 021102 (2004).

[32] P. H. Chavanis, Astron. Astrophys. 356, 1089 (2000).

[33] L. Acedo, Europhys. Lett. 73, 5 (2006).

[34] R. B. Mann and P. Chak, Phys. Rev. E 65, 026128 (2002).

[35] J.-L. Rouet, M. R. Feix, and M. Navet, in Vistas in Astronomy 33, edited by A. Heck (Pergamon, New York, 1990), pp. 357370.

[36] J.-L. Rouet, E. Jamin, and M. R. Feix, in Applying Fractals in
Astronomy, edited by A. Heck and J. M. Perdang (SpringerVerlag, Berlin, 1991), pp. 161-179.

[37] D. Fanelli and E. Aurell, Astron. Astrophys. 395, 399 (2002).

[38] L. A. Kofman, D. Pogosyan, S. F. Shandarin, and A. L. Melott, Astron. J. 393, 437 (1992).

[39] S. F. Shandarin and Y. B. Zeldovich, Rev. Mod. Phys. 61, 185 (1989).

[40] T. Tatekawa and K. Maeda, Astrophys. J. 547, 531 (2001).

[41] M. Vergassola, B. Dubrulle, U. Frisch, and A. Noullez, Astron. Astrophys. 289, 325 (1994).

[42] H. Koyama and T. Konishi, Phys. Lett. A 279, 226 (2001).

[43] H. Koyama and T. Konishi, Europhys. Lett. 58, 356 (2002).

[44] B. N. Miller and J.-L. Rouet, Phys. Rev. E 65, 056121 (2002).

[45] E. Aurell, D. Fanelli, S. N. Gurbatov, and A. Y. Moshkov, Physica D 186, 171 (2003).

[46] J. Binney and S. Tremaine, Galactic Dynamics (Princeton University Press, Princeton, NJ, 1987).

[47] T. C. Halsey, M. H. Jensen, L. P. Kadanoff, I. Procaccia, and B. I. Shraiman, Phys. Rev. A 33, 1141 (1986).

[48] J. Feder, Fractals (Plenum, New York, 1988).

[49] T. Schreiber, Phys. Rev. E 56, 274 (1997).

[50] Y. Shi and C. Gong, Phys. Rev. A 46, 8009 (1992).

[51] P. Levy, Memoir. Sci. Math. Fasc. (Gauthier-Villas, Paris, 1954).

[52] H. Nakao, Phys. Lett. A 266, 282 (2000).

[53] V. Springiel, C. S. Frenk, and S. D. M. White, Nature (London) 440, 1137 (2006).

[54] J. Gaite, Astrophys. J. 658, 11 (2007). 